\title{
A Rising Multiple-Dose Tolerability, Safety, and Pharmacokinetic Study of Intravenous Tildrakizumab in Patients With Psoriasis
}

Anish Mehta ( $\square$ anish.mehta@merck.com )

Merck \& Co., Inc.

Sauzanne Khalilieh ( $\nabla$ sauzanne.khalilieh@merck.com )

Merck \& Co., Inc.

Diana Montgomery

Merck \& Co., Inc.

Anthe Zandvliet

Merck \& Co., Inc.

Xiaoli (Shirley) Hou

Merck \& Co., Inc.

Danlin Xu

Merck \& Co., Inc.

Claus Zachariae

Department of Dermato-allergology, Gentofte Hospital, University of Copenhagen

Thomas Marbury

Orlando Clinical Research Center

Terrill McClanahan

Merck \& Co., Inc.

Wendy Blumenschein

Merck \& Co., Inc.

Harald Kittler

Department of Dermatology, Division of General Dermatology, University of Vienna Medical School, Vienna, Austria.

Ann Horowitz

Merck \& Co., Inc.

\section{Elli Greisenegger}

Department of Dermatology, Division of Immunology, Allergy and Infectious Diseases, University of Vienna Medical School, Vienna, Austria.

\section{Edward Bowman}

Merck \& Co., Inc.

Christine Bangert 
Department of Dermatology, Division of Immunology, Allergy and Infectious Diseases, University of Vienna Medical School, Vienna, Austria.

\section{Elisabeth Riedl}

Department of Dermatology, Division of General Dermatology, University of Vienna Medical School, Vienna, Austria

\section{Tamara Kopp}

Juvenis Medical Center, Vienna, Austria.

\section{Frank van Aarle}

Merck \& Co., Inc.

\section{Method Article}

Keywords: psoriasis; interleukin 23

Posted Date: June 10th, 2015

DOI: https://doi.org/10.1038/protex.2015.025

License: (c) (1) This work is licensed under a Creative Commons Attribution 4.0 International License. Read Full License 


\section{Abstract}

Psoriasis is a chronic inflammatory skin disorder that affects approximately $2-3 \%$ of the population worldwide and has severe effects on patients' physical and psychological well-being1-3. The discovery that psoriasis is an immune-mediated disease has led to more targeted, effective therapies; recent advances have focused on the interleukin \(IL)-12/23p40 subunit shared by IL-12 and IL-23. Evidence suggests that specific inhibition of IL-23 would result in improvement in psoriasis. Here we evaluate tildrakizumab, a monoclonal antibody that targets the IL-23p19 subunit, in a three-part, randomized, placebo-controlled, sequential, rising multiple-dose phase I study in patients with moderate-to-severe psoriasis to provide clinical proof that specific targeting of IL-23p19 results in symptomatic improvement of disease severity in human subjects.

\section{Introduction}

This protocol provides details on the plan and procedures for the following study that was published in Nature: Kopp T, Riedl E, Bangert C, Bowman EP, Greisenegger E, Horowitz A, Kittler H, Blumenschein WM, McClanahan TK, Marbury T, Zachariae C, Xu D, Hou XS, Mehta A, Zandvliet AS, Montgomery D, van Aarle F, Khalilieh S. Clinical improvement in psoriasis with specific targeting of interleukin-23. Nature. 2015 May 14;521 \(7551):222-6. \(doi: 10.1038/nature14175. Epub 2015 Mar 9.) This study evaluates tildrakizumab, a monoclonal antibody that targets the IL-23p19 subunit, in a three-part, randomized, placebo-controlled, sequential, rising multiple-dose phase I study in patients with moderate-to-severe psoriasis to provide clinical proof that specific targeting of IL-23p19 results in symptomatic improvement of disease severity in human subjects.

\section{Reagents}

This was a clinical study with human subjects receiving tildrakizumab $100 \mathrm{mg} / \mathrm{mL}$ injectable intravenous $\backslash$ (IV) solution or placebo \(sterile water for injection placebo added to $0.9 \%$ Saline for IV infusion). See attached protocol for further details.

\section{Equipment}

See attached protocol for full details.

\section{Procedure}

The full protocol is available as an attachment. The following is a summary that is also found in the attached full protocol. Part 1:1) Baseline assessments are taken the evening before dosing 2) Subjects are given study medication after an overnight fast and 2 hours before their first meal. 3) Part 1 includes 4 dose cohorts: cohorts 1 and 2 consist of 4 subjects each, whereas Cohorts 3 and 4 will consist of 8 subjects each. 4) Subjects are randomized at a $3 \backslash$ (active) to $1 \backslash$ (placebo) ratio. 5) Each subject receives 
a single dose of either $0.1 \mathrm{mg} / \mathrm{kg} \backslash$ (Cohort 1 ), $0.5 \mathrm{mg} / \mathrm{kg} \backslash$ (Cohort 2), $3 \mathrm{mg} / \mathrm{kg} \backslash$ (Cohort 3) or $10 \mathrm{mg} / \mathrm{kg} \backslash$ (Cohort 4) of tildrakizumab IV administered over 1 hour; a minimum of 48 hours will elapse between drug administration to subjects. 6) After an 8-week safety observation period, each subject in Part $1 \backslash$ (Cohorts 1 through 4) receives an additional two doses of tildrakizumab IV at $0.1,0.5,3$, and $10 \mathrm{mg} / \mathrm{kg}$, respectively, 4 weeks apart. 7) On Day 2, safety assessments are performed and PK samples obtained. 8) Subjects receive Dose 2 on Day 56 9) Subjects receive Dose 3 on Day 84 10) Subjects have a closeout visit within 14 days of the last safety and PK assessment. Part 21 . Up to 40 subjects with psoriasis are enrolled in Part 2 2. Subjects are put into 2 dose cohorts $\backslash$ (Cohorts 5 and 6 ). 3 . Subjects in Cohort 5 receive 3 doses of $3 \mathrm{mg} / \mathrm{kg}$ tildrakizumab IV given at Week 0,4 , and 8 . 4. Subjects in Cohort 6 receive 3 doses of $10 \mathrm{mg} / \mathrm{kg}$ tildrakizumab IV given at Week 0,4 , and 8 . 5. Subjects are randomized in a 3:1 ratio of active to placebo. 6 . Serum pharmacokinetic samples are obtained predose and at the intervals specified in the attached full protocol 7. Subjects receive Dose 2 and 3 of tildrakizumab during their visits on Days 28 and 56. 8. Subjects have a closeout visit within 14 days of the last PK assessment. 9. A closeout visit will be scheduled within 14 days of the last PK assessment. Skin biopsy, pharmacokinetic, and safety details are provided in the attached full protocol

\section{Anticipated Results}

The primary objectives of this study are to determine the safety, tolerability, and pharmacokinetics of rising intravenous doses of tildrakizumab in subjects with moderate to severe psoriasis. The secondary objectives of this study are to assess the effect of tildrakizumab on skin disease in subjects with moderate to severe psoriasis; and to assess the effects of tildrakizumab on biomarkers of the IL-23 pathway and related immune phenomena.

\section{Supplementary Files}

This is a list of supplementary files associated with this preprint. Click to download.

- supplement0.pdf 